\title{
Exploring the Band Structure of Wurtzite InAs Nanowires Using Photocurrent Spectroscopy
}

Seyyedesadaf Pournia, Samuel Linser, Giriraj Jnawali, Howard E. Jackson and Leigh M. Smith

Department of Physics, University of Cincinnati, Cincinnati, OH 45221-0011

Amira Ameruddin, Philippe Caroff, Jennifer Wong-Leung, Hark Hoe Tan, and Chennupati Jagadish

Department of Electronic Materials Engineering, Research School of Physics, The Australian National University, Canberra, ACT 2601, Australia

Hannah J. Joyce

Department of Engineering, University of Cambridge, $9 \mathrm{JJ}$ Thomson Avenue, Cambridge CB3 OFA, United Kingdom

Address correspondence to leigh.smith@uc.edu

ABSTRACT:

We use polarized photocurrent spectroscopy in a nanowire device to investigate the band structure of hexagonal Wurtzite InAs. Signatures of optical transitions between four valence bands and two conduction bands are observed which are consistent with the symmetries expected from group theory. The ground state transition energy identified from photocurrent spectra is seen to be consistent with photoluminescence emitted from a cluster of nanowires from the same growth substrate. From the energies of the observed bands we determine the spin orbit and crystal field energies in Wurtzite InAs. This information is essential to the development of crystal phase engineering of this important III-V semiconductor.

Keywords: Nanowires, Wurtzite InAs, Photocurrent Measurement, Optical Selection Rules, Energy Band Structure. 


\section{Introduction}

III-V semiconductor nanowires (NWs) are quasi-one-dimensional materials which show great promise as a nanoscale platform for efficient and high-speed electronic devices, nanosensors and photovoltaics[1,2]. Among the III-V NWs, InAs is of particular interest because it exhibits a high electron mobility, a low effective electron mass, a large spin orbit energy and a small energy band gap. The spin-orbit energy is nearly as large as the band gap energy resulting in strong momentum-spin coupling which has been utilized in the search for Marjarona fermions[3]. Like many III-V NWs, InAs occurs in both the usual Zincblende (ZB) cubic phase, but also hexagonal Wurtzite (WZ). The hexagonal Wurtzite crystal structure has a lower symmetry than the cubic Zincblende structure which has a large impact on both the band structure and selection rules for optical transitions. The resulting differences in the band structure have been extensively explored for both $\operatorname{InP}[4,5,6,7]$ and GaAs $[8,9] \mathrm{NWs}$. This detailed understanding has resulted in the rapid development of ZB/WZ nanowire axial and radial heterostructures in these materials to control the thermal conductivity [10], g-factor and diamagnetic coefficients $[11,12,13]$, and enhanced control of emission quantum efficiencies and detector sensitivities $[14,15,16,17,18]$. There is also intense interest in developing hexagonal Si, Ge and SiGe alloys as direct-gap materials forming the basis for silicon-based optoelectronics $[19,20,21]$.

Much less is known about Wurtzite InAs. Experimental measurements of the WZ InAs fundamental gap range from $0.43 \mathrm{eV}$ to $0.54 \mathrm{eV}[22,23,24,25]$ and theoretical calculations of the gap range from 0.46 to $0.481 \mathrm{eV}[26,27,28,29,30]$. There are no direct measures of the 
valence and conduction band structure, but a number of theoretical calculations exist $[26,27,28$ ,29,30]. In this letter, we use polarized photocurrent spectroscopy in a WZ NW device to determine its band structure over a wide range of energies from 0.3 to $1.2 \mathrm{eV}$.

\section{Material and Methods}

Wurtzite InAs nanowires were fabricated on a [111]-oriented InAs substrate using the metal-catalyzed MOCVD (metal-organic chemical vapor deposition) growth method with $50 \mathrm{~nm}$ gold nanoparticles. A substrate temperature of $500^{\circ} \mathrm{C}$ and an arsine/trimethylindium ratio of 2.9 were used to achieve WZ crystal growth [31]. Fig. 1 (a) and (b) show a SEM image of the growth substrate and two plan view HRTEM images of a typical nanowire demonstrating the single phase WZ nature of the nanowires. The NWs were removed mechanically from the growth substrate into a methanol solution and dispersed on a $\mathrm{p}$-doped silicon substrate with a $300 \mu \mathrm{m} \mathrm{SiO}_{2}$ layer on the surface. The dispersed wires were usually small $(<5)$ bundles of wires that formed through van der Waals forces. A well-separated bundle of wires was identified using optical microscopy for device fabrication. To fabricate the device, two $20 \mathrm{~nm}$ Titanium and $300 \mathrm{~nm}$ Aluminum contacts were deposited on either end of the wire using photolithography, metal deposition and liftoff. After confirmation that the contacts were conductive using a probe station, the nanowire device was placed into a gold-plated chip carrier which was mounted onto the cold finger of an optical cryostat for low temperature measurements.

Figure 2 shows a typical I-V measurement on a $50 \mathrm{~nm}$ nanowire device at room temperature, in the dark and under white light illumination. From this measurement, we 
estimate the resistivity of the nanowire to be $0.9 \Omega . \mathrm{mm}$. Assuming an electron mobility of 210 $\mathrm{cm}^{2} \cdot \mathrm{V}^{-1} \cdot \mathrm{s}^{-1}$ (measured in similarly synthesized samples [32]), one can estimate that the carrier density in the nanowire to be $n \sim 3 \times 10^{17} \mathrm{~cm}^{-3}$ which is also consistent with Ref. [33]. Photocurrent spectra were obtained by fixing the current in the device and measuring the change in the bias voltage as the laser is tuned over a wavelength range. The laser is mechanically chopped and the change in the bias voltage is measured using a lock-in amplifier.

3. Background:

For ZB NWs, optical transitions which promote electrons from the valence bands to the conduction bands are allowed for any polarization of light. The selection rules for optical transitions between the various valence bands and conduction bands in WZ NWs are more complicated. WZ InAs belongs to the $C_{6 v}$ point-group symmetry (cubic Zincblende InAs has $T_{d}$ symmetry). The valence bands at the center of the Brillouin zone can be ordered from highest energy to lowest energy as first $(A)$, second $(B)$, third (C), and fourth (D) valence bands with $\Gamma_{9}$, $\Gamma_{7}, \Gamma_{7}$, and $\Gamma_{9}$ symmetries, respectively (see Fig. 1). Similarly, the lowest lying conduction band $\left(\mathrm{CB}_{1}\right)$ has $\Gamma_{7}$ symmetry, and a second conduction band $\left(\mathrm{CB}_{2}\right)$ at higher energies with $\Gamma_{8}$ symmetry which results from zone folding of the L-valley in the cubic Zincblende structure to the center of the Brillouin zone $(k=0)$ in the Wurtzite phase [26].

To determine the optical selection rules for the $C_{6 v}$ symmetry group, the light polarized perpendicular $(E \perp C)$ and parallel (Ellc) to the long axis of the nanowire (the Wurtzite c-axis) are associated with $\Gamma_{5}$ and $\Gamma_{1}$ symmetries, respectively ${ }^{34}$. An optical transition is allowed for a particular polarization if the product of the initial and final state symmetries contains the 
symmetry associated with a particular polarization. In equation (1) below, the various band symmetries are multiplied, and the results tabulated.

$$
\begin{gathered}
\Gamma_{9}(A, D) \times \Gamma_{7}(C B 1)=\Gamma_{5}(E \perp C)+\Gamma_{6} \\
\Gamma_{7}(B, C) \times \Gamma_{7}(C B 1)=\Gamma_{1}(E I I C)+\Gamma_{6}+\Gamma_{5}(E \perp C) \\
\Gamma_{9}(A, D) \times \Gamma_{8}(C B 2)=\Gamma_{5}(E \perp c)+\Gamma_{6} \\
\Gamma_{7}(B, C) \times \Gamma_{8}(C B 2)=\Gamma_{3}+\Gamma_{4}+\Gamma_{6}
\end{gathered}
$$

From equation (1), we see that for hexagonal Wurtzite InAs, optical transitions from the A or D valence bands to $\mathrm{CB}_{1}$ are only allowed for perpendicular polarization, while optical transitions from the $\mathrm{B}$ or $\mathrm{C}$ valence bands to $\mathrm{CB}_{1}$ are allowed for both polarizations. Perpendicularly polarized light can excite carriers from the A or D valence bands to the second conduction band, however, excitations from either $B$ or $C$ valence bands to $\mathrm{CB}_{2}$ are not allowed at all.

Figure 1 provides a summary of all the allowed optical transitions in the $C_{6 v}$ symmetry group.

\section{Experiment and Results:}

In this letter we explore the energy band structure and optical selection rules in a WZ

InAs NW device by using polarized photocurrent (PC) spectroscopy in the infrared energy range

0.3-1.2 eV (or 4000 to $1000 \mathrm{~nm}$ ) at $300 \mathrm{~K}$ and $10 \mathrm{~K}$. The signal and idler output from an OPO pumped by a $4 \mathrm{~W}$ Ti-sapphire laser is continuously tuned from 0.3 to $1.2 \mathrm{eV}$. The polarization is 
made linear by using a wire grid polarizer and then rotated using a $\mathrm{CaF}_{2}$ double Fresnel Rhomb rotator to align parallel or perpendicular to the nanowire device. The laser beam was attenuated to an average power of $200 \mathrm{uW}$ and focused onto the sample using a $40 \mathrm{X}$ reflective objective to a 1.3-5 micron spot varying with the wavelength of the laser. Representative PC spectra are shown at both $10 \mathrm{~K}$ and $300 \mathrm{~K}$ in Fig. 3. Errors associated with each transition are estimated by at least two or more measurements taken at different times on the same device as shown in the Supplemental Information. For the PC spectrum taken at $10 \mathrm{~K}$, we see that there is no photocurrent at low energies and a clear onset at $0.473 \pm 0.002 \mathrm{eV}$ eV for light polarized perpendicularly to the NW long axis (c-axis), with a peak appearing at $0.482 \pm$ $0.006 \mathrm{eV}$ signaling the transition from the $A$ valence band to $\mathrm{CB}_{1}$.

For comparison, photoluminescence (PL) measurements were taken on clusters of wires dispersed onto a silicon substrate from the same growth by using $800 \mathrm{~nm}$ excitation as an excitation source. The laser was chopped at $300 \mathrm{~Hz}$ and focused onto the nanowire cluster using a 40X/0.5 NA reflective objective. PL emitted by the NW cluster was collected by the same objective and focused onto the entrance slit of a $0.2 \mathrm{~m}$ spectrometer and dispersed by a 600 line/mm grating. The PL was detected by a lock-in and an InSb pn diode cooled to $77 \mathrm{~K}$. Below the $10 \mathrm{~K}$ PC spectra, we display $10 \mathrm{~K} \mathrm{PL}$ spectrum taken from a cluster of approximately ten similar nanowires, which confirms the assignment of the onset and peak from the PC 
spectrum. The fundamental band gap for Wurtzite material is thus 60 meV higher than Zincblende InAs band gap as expected from both theory and recent experiments $22,23,24,25,29,30$.

The photocurrent spectrum for light polarized parallel to the NW (c axis) shows a higher energy onset and peaks at $0.550 \pm 0.005 \mathrm{eV}$ which is assumed to be the excitation from the $\mathrm{B}$ valence band to $\mathrm{CB}_{1}$. The peak at $0.915 \pm 0.005 \mathrm{eV}$ which is visible for both parallel and perpendicular polarizations is assigned to the transition from the third valence band $(\mathrm{C})$ to the first conduction band $\left(\mathrm{CB}_{1}\right)$.

At higher energies, two additional but weak peaks are observed only for perpendicular excitation at $0.988 \pm 0.003 \mathrm{eV}$ and $1.065 \pm 0.004 \mathrm{eV}$. Based on the selection rules described previously, we find these transitions can result from transitions either from $A$ to $C B_{2}$, or $D$ to $\mathrm{CB}_{1}$. In order to most closely match De and Pryor's calculation for the splitting between $\mathrm{CB}_{1}$ and $\mathrm{CB}_{2}$ and the splitting between $C$ and $\mathrm{D}$, we assign the $1.065 \mathrm{eV}$ transition to $A$ to $\mathrm{CB}_{2}$ and the $0.988 \mathrm{eV}$ transition to $\mathrm{D}$ to $\mathrm{CB}_{1}{ }^{26}$. From this assignment, we find the splitting between $\mathrm{CB}_{2}$ and $\mathrm{CB}_{1}$ to be $0.583 \pm 0.010 \mathrm{eV}$ (De and Pryor predict a splitting of $0.74 \mathrm{eV}$ ). We find the splitting between the $\mathrm{D}$ and $\mathrm{C}$ valence bands to be $0.073 \pm 0.008 \mathrm{eV}$ (De and Pryor predict $0.183 \mathrm{eV}$ ). The energy gap and transition energies and energy splittings measured in our experiment are shown in Table 1 and compared with both theoretical calculations and experiments. 
Photocurrent spectra taken at $300 \mathrm{~K}$ (see Fig. 3) shows the first three transitions obeying the expected selection rules, but shifted towards lower energy because of the temperaturedependent shift of the energy gap $[35,36,37]$. The observed energy splitting between valence bands exhibits no obvious temperature dependence between the $10 \mathrm{~K}$ and $300 \mathrm{~K}$ measurements, consistent with results in the other material [38].

Using the $A B$ and $A C$ splittings obtained from these measurements, it is possible to extract the spin-orbit energy and crystal field energy for this structure using the quasi-cubic approximation $[26,39]$ shown in equation 2 , below:

$$
\Delta_{S O, C F}=\frac{1}{2}\left(\Delta_{A B}+\Delta_{A C} \pm \sqrt{\Delta_{A B}^{2}+\Delta_{A C}^{2}-4 \Delta_{A B} \Delta_{A C}}\right)
$$

As noted in several publications equation 2 results in two energies which may be assigned to either the SO or the CF energies $[6,26,34]$. The spin-orbit energy results from the constituent In and As atoms in these materials so one would expect very similar spin-orbit energies for either Wurtzite or Zincblende InAs whose spin-orbit energy is $0.38 \mathrm{eV}[40,41]$. Equation 2 provides the two solutions $0.387 \mathrm{eV}$ and $0.113 \mathrm{eV}$, for the crystal splitting or spin-orbit splitting. It seems reasonable to assign $0.387 \mathrm{eV}$ to the $W Z$ InAs spin orbit energy and $0.113 \mathrm{eV}$ to the crystal field energy. These results are tabulated in Table 1 for both $300 \mathrm{~K}$ and $10 \mathrm{~K}$ measurements. 
TABLE 1: The energy bands splitting from the experiments at $T=10 \mathrm{~K}$ and $\mathrm{T}=300 \mathrm{~K}$

\begin{tabular}{|c|c|c|c|c|c|c|c|c|}
\hline & \multicolumn{7}{|c|}{ Wurtzite InAs } & \\
\cline { 2 - 8 } & $\mathrm{E}_{\mathrm{g}}(\mathrm{eV})$ & $\Delta_{\mathrm{AB}}(\mathrm{eV})$ & $\Delta_{\mathrm{AC}}(\mathrm{eV})$ & $\Delta_{\mathrm{AD}}(\mathrm{eV})$ & $\Delta_{\mathrm{CB} 1-\mathrm{CB} 2}(\mathrm{eV})$ & $\Delta_{\mathrm{SO}}(\mathrm{eV})$ & $\Delta_{\mathrm{CF}}(\mathrm{eV})$ & $\begin{array}{c}\Delta\left(\mathrm{E}_{\mathrm{g}}{ }^{\mathrm{WZ}}-\mathrm{E}_{\mathrm{g}}{ }^{\mathrm{ZB}}\right) \\
(\mathrm{eV})\end{array}$ \\
\hline $\mathrm{T}=300 \mathrm{~K}$ & 0.438 & 0.060 & 0.426 & - & - & 0.387 & 0.098 & 0.7 \\
\hline $\mathrm{T}=10 \mathrm{~K}$ & 0.482 & 0.068 & 0.433 & 0.505 & 0.583 & 0.387 & 0.113 & 0.6 \\
\hline
\end{tabular}

\section{Discussion}

We now compare the results from our measurements to both theoretical calculations of the WZ InAs band structure, and other experimental measurements of the fundamental gap. Results from the $10 \mathrm{~K}$ data is compared with the other theoretical and experimental results in Table 2. De and Pryor calculated the WZ band structure for InAs using empirical pseudopotentials [26]. Junior et al calculated the WZ InAs band structure using Density Functional Theorey (DFT) with a modified Becke-Johnson (mBJ) exchange potential with Local Density Approximation (LDA) correlations [27]. Gimitra and Fabian use DFT with semilocal modified Becke-Johnson exchange potentials (TB-mBJ) and with LDA correlations [28]. 
Bechstedt and Belabbes used DFT with LDA exchange and correlations to calculate the band structure of the various InAs polytypes, including pure WZ [30]. Zanolli et al used DFT calculations using LDA exchange and correlations followed by GW corrections [29].

The parameters for the various $A B$ and $A C$ valence band splittings, and splittings between the two conduction bands, along with SO and CF energies are tabulated from these different calculations in Table 2 and compared with the results here. While all of the theoretical calculations of the fundamental gap agree fairly closely with each other and also with our present measurement, there is substantial variability for the other parameters. For example, theoretical estimates of the $A B$ valence band splitting range from 59 to $105 \mathrm{meV}$ as compared with our measurement of $70 \mathrm{meV}[26,27,28]$. The theoretical AC valence band splitting ranges from 350 to $470 \mathrm{meV}$ as compared with our measurement of $440 \mathrm{meV}$ $[26,27,28]$. The theoretical crystal field energies range from 95 to 195 meV as compared with our measurement of $120 \mathrm{meV}[26,30]$. The theoretical spin orbit energies range from 356 to $379 \mathrm{meV}$ while our measurement is $390 \mathrm{meV}[26,30]$. The largest discrepancy is seen in our measurement of the CB splittings to be 590 meV with theoretical estimates of the CB splitting substantially higher at 706 to $741 \mathrm{meV}[26,28]$.

TABLE 2: Comparison between the experiment results at $\mathrm{T}=10 \mathrm{~K}$ and other experimental and theoretical studies 


\begin{tabular}{|c|c|c|c|}
\hline & Theory & Other experiments & $\begin{array}{l}\text { PC measurement in } \\
\text { figure } 3(10 \mathrm{~K})\end{array}$ \\
\hline$E_{g}(e V)$ & $\begin{array}{l}0.47^{29} 0.46^{28} \\
0.481^{30,26} 0.467^{27}\end{array}$ & $\begin{array}{l}0.52(7 K)^{23} 0.477(11 K)^{22} \\
0.458(5 K)^{24} 0.54(5 K)^{25} \\
0.43(10 K)^{42}\end{array}$ & 0.482 \\
\hline$\Delta_{\mathrm{AB}}(\mathrm{eV})$ & $\begin{array}{l}0.066^{28} 0.105^{26} \\
0.0592^{27}\end{array}$ & & 0.068 \\
\hline$\Delta_{\mathrm{AC}}(\mathrm{eV})$ & $\begin{array}{l}0.36^{28} 0.469^{26} \\
0.3527^{27}\end{array}$ & & 0.433 \\
\hline$\Delta_{\mathrm{CB} 1-\mathrm{CB} 2}(\mathrm{eV})$ & $0.706^{28} 0.741^{26}$ & & 0.583 \\
\hline$\Delta_{\mathrm{CF}}(\mathrm{eV})$ & $0.095^{30} 0.195^{26}$ & & 0.113 \\
\hline$\Delta_{\mathrm{so}}(\mathrm{eV})$ & $0.356^{30} 0.379^{26}$ & & 0.387 \\
\hline
\end{tabular}

In order to gauge the strength of the polarization selection rules we now look at the degree of polarization for these different transitions. We define the degree of polarization as DOP $=\left(I_{\perp}-I_{I I}\right) /\left(I_{\perp}+I_{I I}\right)$ to describe the polarization dependence of the photocurrent spectra. Based on classical electrodynamics, if a cylinder with dielectric constant $\varepsilon$, is placed in an electric field $\mathrm{E}$, the component of the internal field parallel to the axis of cylinder is the same as the outside field $\left(E_{\| 1}=E_{011}\right)$, but it is attenuated in the perpendicular direction $\left(E_{1}=\frac{2}{1+} E_{0_{1}}\right)$ [43]. Since the absorbed energy is $\propto E^{2}$, for an InAs wire with $\varepsilon=16.78$, for light polarized perpendicular to the NW with an intensity of $P_{0}$ the intensity of light inside the nanowire would be $P_{0} / 79$. Similarly, for light polarized parallel to the NW with an intensity $\mathrm{P}_{0}$ the intensity inside the nanowire would also be $P_{0}$. The degree of polarization in our nanowire is plotted in Fig. 4 for the $10 \mathrm{~K}$ and $300 \mathrm{~K}$ measurements. A sharp peak centered at $0.48 \mathrm{eV}$ is observed, showing an $85 \%$ perpendicularly polarized photocurrent around the fundamental band gap energy. 
The response of the device to polarized light is a rather complicated combination of the dielectric response of the nanowire itself, and also the optical selection rules. We attempt to disentangle these responses by using the dielectric response derived above. Assuming that the incident power on the nanowire is the same for parallel and perpendicular polarizations, this can be expressed as [44]:

$$
\frac{I \perp}{I \|}=\left(\frac{2}{1+\varepsilon \perp}\right)^{2} \frac{\alpha \perp}{\alpha \|}
$$

Where $\varepsilon \perp$ is the real part of the dielectric constant in the direction perpendicular to the $\mathrm{c}$ axis of wire and $\alpha \perp$ is the absorption constant. Since the $\alpha \perp$ is directly proportional to the imaginary part of the dielectric constant $\left(\varepsilon^{\prime \prime}\right)[45]$ and inversely proportional to the index of refraction, and we know that $\varepsilon^{\prime \prime}$ is proportional to the oscillator strength (f), we find the perpendicular to parallel current ratio to be equal to:

$$
\frac{I \perp}{I \|}=\left(\frac{2}{1+\varepsilon \perp}\right)^{2} \frac{f \perp}{f \|} \sqrt{\frac{\varepsilon \|}{\varepsilon \perp}}
$$

From our measurement, the ratio of the polarized currents is $\sim 12.5$ at the $A \rightarrow C B 1$ transition. By using the dielectric constants for light parallel and perpendicular to the c-axis calculated by De and Pryor $\left(\frac{\varepsilon \|}{\varepsilon \perp} \sim 0.81\right)$ [46], we find $\frac{f \perp}{f \|} \sim 1100$, which shows that excitation of a dipole perpendicular to the nanowire has a much stronger oscillator strength than parallel. This 
implies that the absorption of parallel polarized light for this lowest energy transition is nearly negligible as anticipated from group theory (shown previously) [47,48].

\section{Summary and Conclusions}

Using polarized PC spectroscopy over the energy range 0.3 to $1.2 \mathrm{eV}$, we have identified the energies of four valence and two conduction bands in Wurtzite InAs nanowires. The results agree well with predicted energy gaps from empirical pseudopotential calculations and the polarization of the peaks are in excellent agreement with the optical selection rules for a hexagonal material. From the $A B$ and $A C$ valence band splittings, we extract a spin-orbit energy of $0.38 \mathrm{eV}$ and a crystal field energy of $0.12 \mathrm{eV}$ for Wurtzite InAs. The splitting between the first and second conduction bands is observed to be $0.59 \mathrm{eV}$. From the degree of polarization measured from the $A$ to $C B 1$ ground state transition we show that the ratio of the perpendicular to parallel oscillator strength is $\sim 1100$. Using these band energies, it should now be possible to optimize ab initio band structure calculations. Detailed understanding of the WZ InAs band structure will be essential to the development of crystal phase engineering of this important infrared optoelectronic and high speed electronic materials system.

\section{Acknowledgements}

We acknowledge the financial support of the NSF through Grants DMR 1507844, DMR 1531373, and ECCS 1509706 and also the financial support of the Australian Research Council and the European Research Council (Grant No. 716471, ACrossWire).. The Australian National Fabrication Facility (ACT Node) is acknowledged for access to the growth facility used in this work. The Australian Microscopy and Microanalysis Research Facility is acknowledged for 
access to the electron microscopes used in this work.
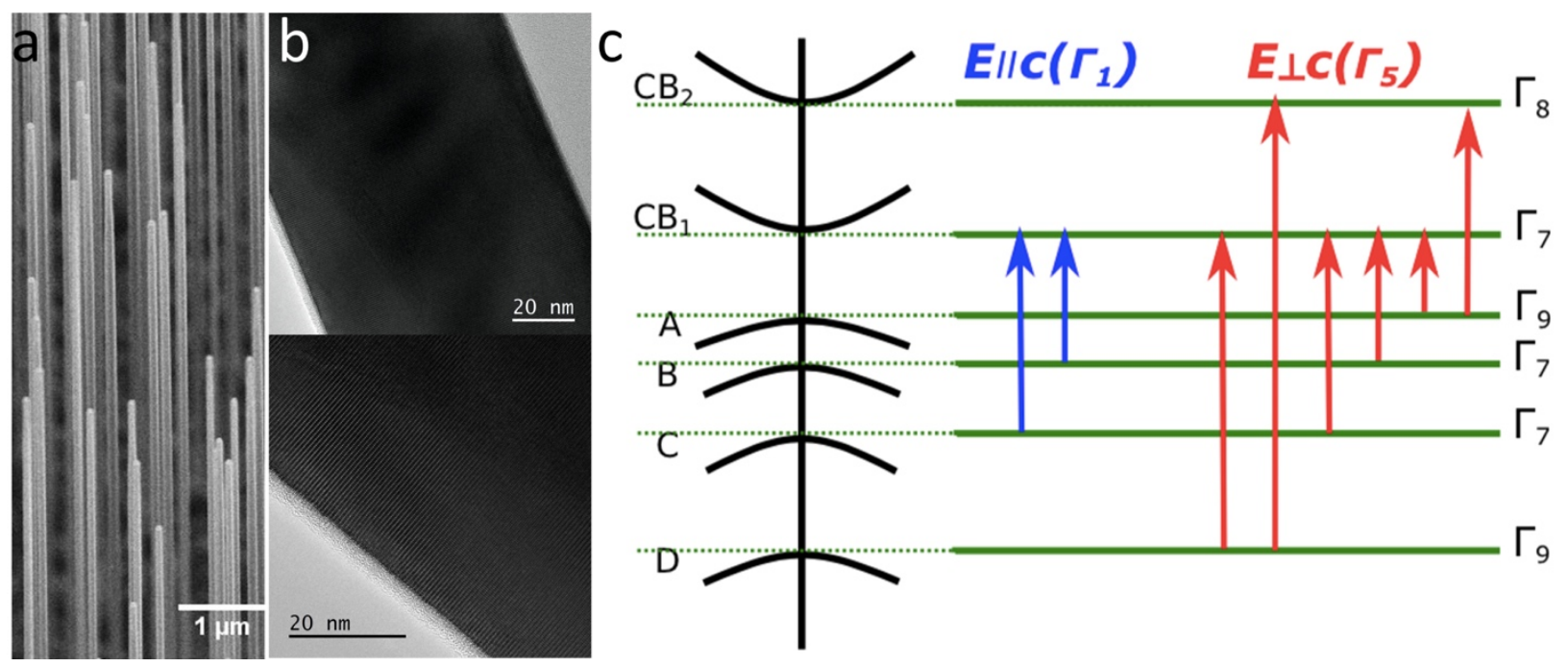

Figure 1.(a) The SEM image and (b) the TEM of $50 \mathrm{~nm}$ diameter Wurtzite InAs nanowires on the initial substrate. (c) shows the band diagram of Wurtzite structure and the allowed optical transitions from four of the valence band $(A, B, C, D)$ and The two conduction bands (CB1 and CB2) considering their symmetries. 


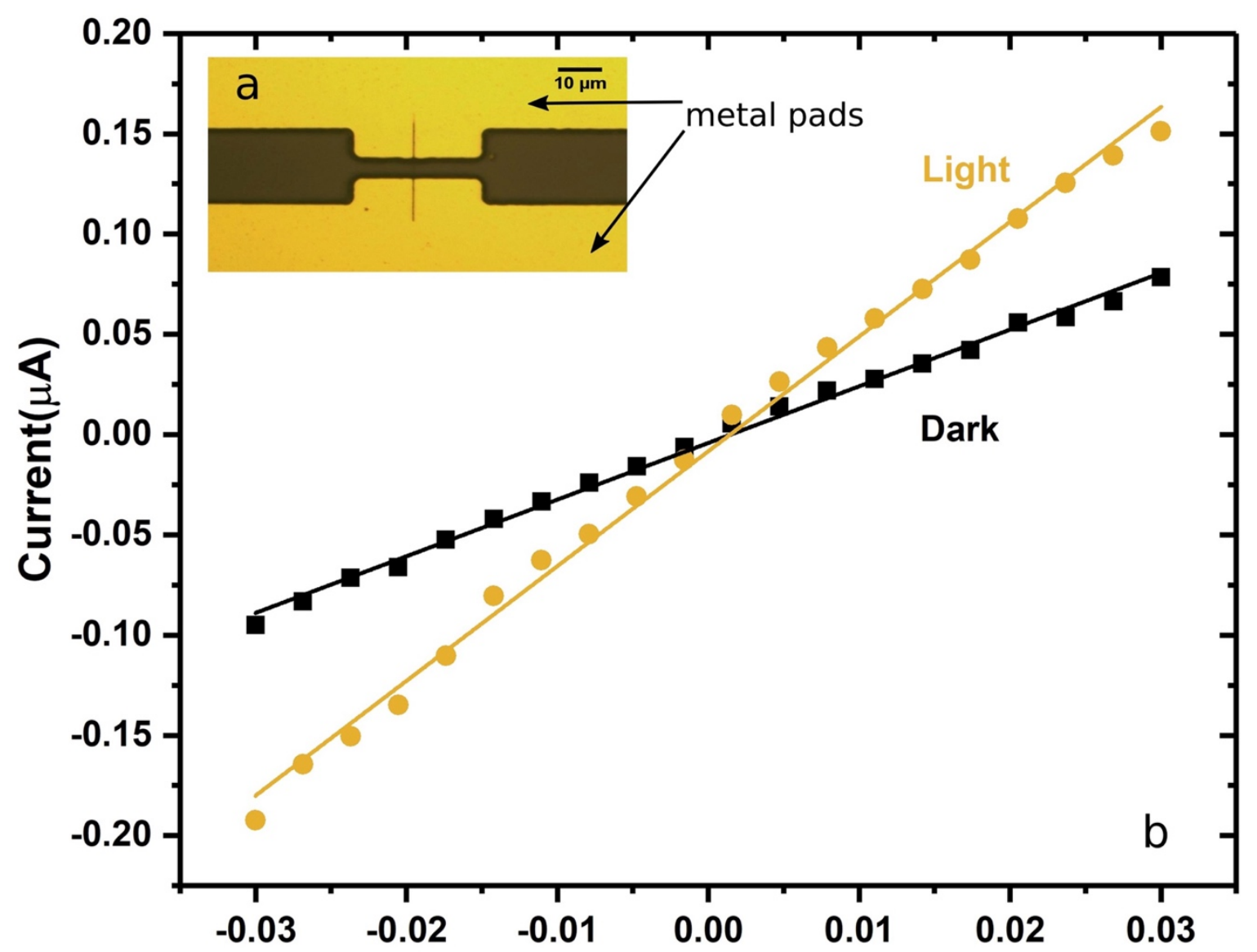

Figure 2.IV characteristics of Wurtzite InAs nanowire device obtained in the dark and under white light illumination at 300K. Inset shows optical image of the nanowire device with Ti/Al pads. 


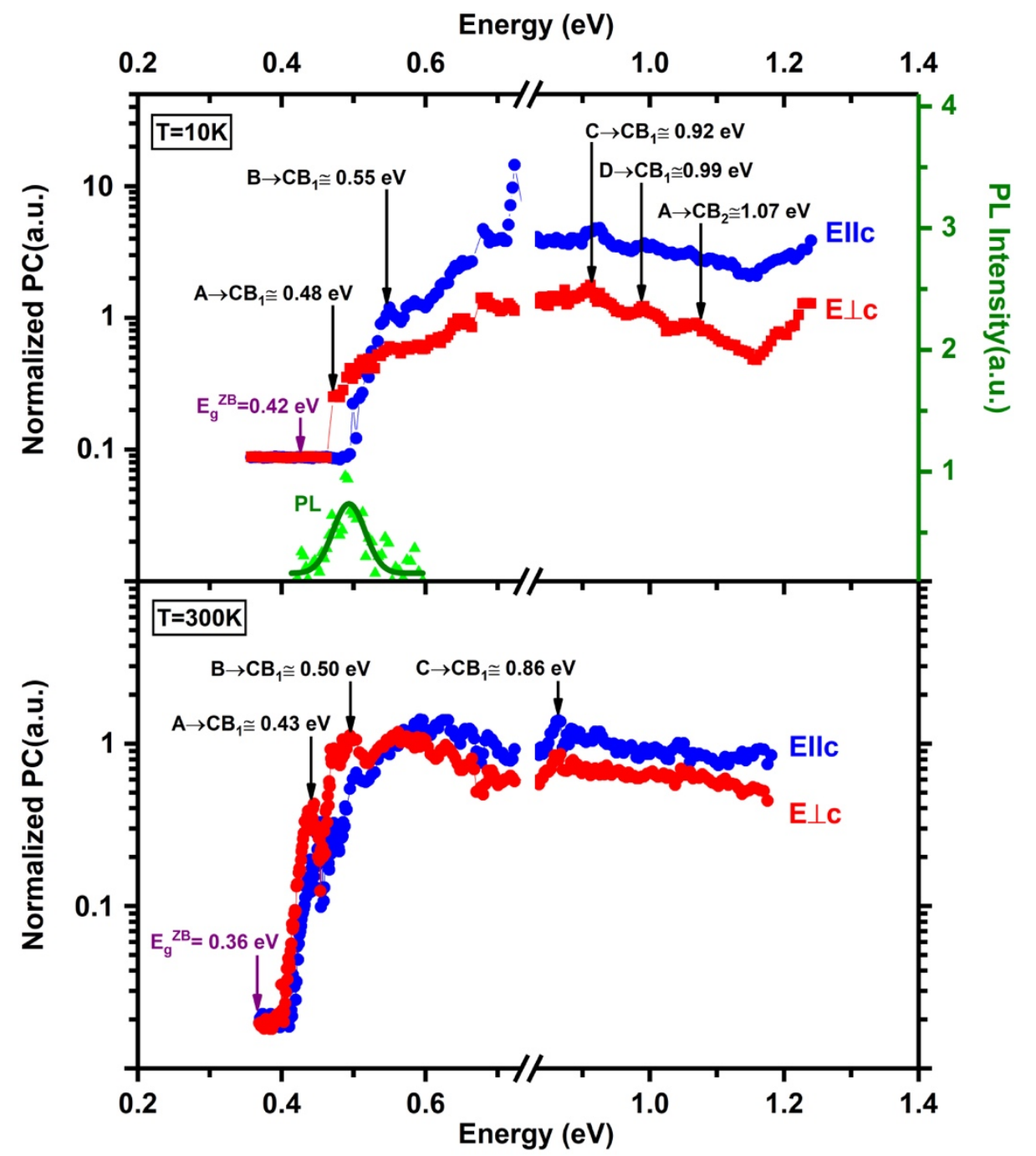

Figure 3.The photocurrent spectra of a Wurtzite InAs nanowire acquired by exciting the carriers with polarization perpendicular to the nanowire (red squares) and parallel to the nanowire (blue circles) at temperature $300 \mathrm{~K}$ (on the top) and $10 \mathrm{~K}$ (bottom). The unpolarized PL spectra taken from a nanowire mechanically transferred on a Si substrate, shown by the green triangles and the fit solid line, confirms the fundamental bandgap at 10K. The energies of the peaks representing different excitations are marked on the diagram. The peaks of the higher temperature measurement are shifted to lower energies in comparison with $10 \mathrm{~K}$ measurement, due to increased thermal energy and higher interatomic spacing. 


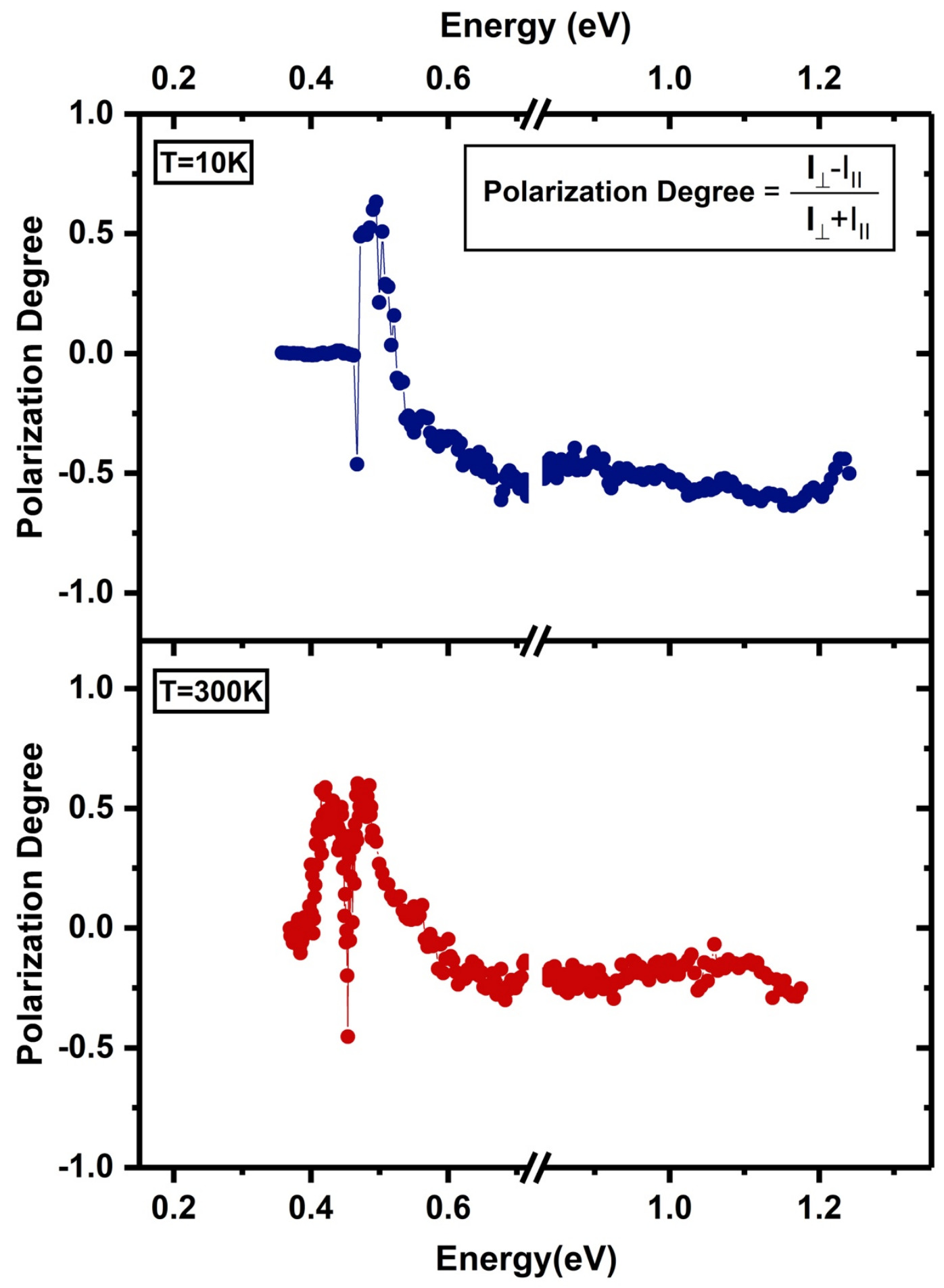

Figure 4.Polarization of the photocurrent spectra at $10 \mathrm{~K}$ and $300 \mathrm{~K}$ calculated from data shown in Fig. 3. 


\section{References:}

(1) Editors, G.; Nocera, D.; Guldi, D. 2009 Renewable Energy Issue. Chemical Society Reviews 2009, 38 (1), 165-184. https://doi.org/10.1039/b800582f.

(2) LaPierre, R. R. III-V Nanowire Solar Cells. Optics InfoBase Conference Papers 2011. https://doi.org/10.1146/annurev-matsci-062910-100434.

(3) Das, A.; Ronen, Y.; Most, Y.; Oreg, Y.; Heiblum, M.; Shtrikman, H. Zero-Bias Peaks and Splitting in an Al-InAs Nanowire Topological Superconductor as a Signature of Majorana Fermions. Nature Physics 2012, 8 (12), 887-895. https://doi.org/10.1038/nphys2479.

(4) Mishra, A.; Titova, L. V.; Hoang, T. B.; Jackson, H. E.; Smith, L. M.; Yarrison-Rice, J. M.; Kim, Y.; Joyce, H. J.; Gao, Q.; Tan, H. H.; et al. Polarization and Temperature Dependence of Photoluminescence from Zincblende and Wurtzite InP Nanowires. Applied Physics Letters 2007, 91 (26), 89-92. https://doi.org/10.1063/1.2828034.

(5) Pemasiri, K.; Jackson, H. E.; Smith, L. M.; Wong, B. M.; Paiman, S.; Gao, Q.; Tan, H. H.; Jagadish, C. Quantum Confinement of Excitons in Wurtzite InP Nanowires. Journal of Applied Physics 2015, 117 (19). https://doi.org/10.1063/1.4921109.

(6) Perera, S.; Shi, T.; Fickenscher, M. A.; Jackson, H. E.; Smith, L. M.; Yarrison-Rice, J. M.; Paiman, S.; Gao, Q.; Tan, H. H.; Jagadish, C. Illuminating the Second Conduction Band and Spin-Orbit Energy in Single Wurtzite InP Nanowires. Nano Letters 2013, 13 (11), 53675372. https://doi.org/10.1021/nl4028878.

(7) Wallentin, J.; Mergenthaler, K.; Ek, M.; Wallenberg, L. R.; Samuelson, L.; Deppert, K.; Pistol, M. E.; Borgstroöm, M. T. Probing the Wurtzite Conduction Band Structure Using State Filling in Highly Doped InP Nanowires. Nano Letters 2011, 11 (6), 2286-2290. https://doi.org/10.1021/nl200492g.

(8) Khomyakov, P. A.; Karg, S.; Dheeraj, D. L.; Gotsmann, B.; Weman, H.; Riel, H.; Signorello, G.; Lo, E. Uniaxial Stress. nature communication 2014, No. 7491. https://doi.org/10.1038/ncomms4655.

(9) Ahtapodov, L.; Todorovic, J.; Olk, P.; Mjåland, T.; Slåttnes, P.; Dheeraj, D. L.; Van Helvoort, A. T. J.; Fimland, B. O.; Weman, H. A Story Told by a Single Nanowire: Optical Properties of Wurtzite GaAs. Nano Letters 2012, 12 (12), 6090-6095. https://doi.org/10.1021/nl3025714.

(10) Raya-Moreno, M.; Rurali, R.; Cartoixà, X. Thermal Conductivity for III-V and II-VI Semiconductor Wurtzite and Zinc-Blende Polytypes: The Role of Anharmonicity and Phase Space. Physical Review Materials 2019, 3 (8), 1-13. https://doi.org/10.1103/physrevmaterials.3.084607.

(11) Wu, S.; Peng, K.; Battiato, S.; Zannier, V.; Bertoni, A.; Goldoni, G.; Xie, X.; Yang, J.; Xiao, S.; Qian, C.; et al. Anisotropies of the G-Factor Tensor and Diamagnetic Coefficient in Crystal-Phase Quantum Dots in InP Nanowires. Nano Research 2019, 12 (11), 11-12. https://doi.org/10.1007/s12274-019-2522-5.

(12) Faria Junior, P. E.; Tedeschi, D.; De Luca, M.; Scharf, B.; Polimeni, A.; Fabian, J. Common Nonlinear Features and Spin-Orbit Coupling Effects in the Zeeman Splitting of Novel 
Wurtzite Materials. Physical Review B 2019, 99 (19), 1-9.

https://doi.org/10.1103/PhysRevB.99.195205.

(13) Tedeschi, D.; De Luca, M.; Faria Junior, P. E.; Granados Del Águila, A.; Gao, Q.; Tan, H. H.; Scharf, B.; Christianen, P. C. M.; Jagadish, C.; Fabian, J.; et al. Unusual Spin Properties of InP Wurtzite Nanowires Revealed by Zeeman Splitting Spectroscopy. Physical Review B 2019, 99 (16), 161204. https://doi.org/10.1103/PhysRevB.99.161204.

(14) Yuan, X.; Li, L.; Li, Z.; Wang, F.; Wang, N.; Fu, L.; He, J.; Tan, H. H.; Jagadish, C. Unexpected Benefits of Stacking Faults on the Electronic Structure and Optical Emission in Wurtzite GaAs/GalnP Core/Shell Nanowires. Nanoscale 2019, 11 (18), 9207-9215. https://doi.org/10.1039/c9nr01213c.

(15) Zhou, C.; Zhang, X. T.; Zheng, K.; Chen, P. P.; Matsumura, S.; Lu, W.; Zou, J. Epitaxial GaAs/AlGaAs Core-Multishell Nanowires with Enhanced Photoluminescence Lifetime. Nanoscale 2019, 11 (14), 6859-6865. https://doi.org/10.1039/c9nr01715a.

(16) Spies, M.; Monroy, E. Nanowire Photodetectors Based on Wurtzite Semiconductor Heterostructures. Semiconductor Science and Technology 2019, 34 (5). https://doi.org/10.1088/1361-6641/ab0cb8.

(17) Battiato, S.; Wu, S.; Zannier, V.; Bertoni, A.; Goldoni, G.; Li, A.; Xiao, S.; Han, X. D.; Beltram, F.; Sorba, L.; et al. Polychromatic Emission in a Wide Energy Range from InPInAs-InP Multi-Shell Nanowires. Nanotechnology 2019, 30 (19). https://doi.org/10.1088/1361-6528/aafde4.

(18) Göransson, D. J. O.; Borgström, M. T.; Huang, Y. Q.; Messing, M. E.; Hessman, D.; Buyanova, I. A.; Chen, W. M.; Xu, H. Q. Measurements of Strain and Bandgap of Coherently Epitaxially Grown Wurtzite InAsP-InP Core-Shell Nanowires. Nano Letters 2019, 19 (4), 2674-2681. https://doi.org/10.1021/acs.nanolett.9b00644.

(19) Ren, Y.; Leubner, P.; Verheijen, M. A.; Haverkort, J. E. M.; Bakkers, E. P. A. M. Hexagonal Silicon Grown from Higher Order Silanes. Nanotechnology 2019, 30 (29). https://doi.org/10.1088/1361-6528/ab0d46.

(20) Cartoixà, X.; Palummo, M.; Hauge, H. I. T.; Bakkers, E. P. A. M.; Rurali, R. Optical Emission in Hexagonal SiGe Nanowires. Nano Letters 2017, 17 (8), 4753-4758. https://doi.org/10.1021/acs.nanolett.7b01441.

(21) Dixit, S.; Shukla, A. K. Optical Properties of Lonsdaleite Silicon Nanowires: A Promising Material for Optoelectronic Applications. Journal of Applied Physics 2018, 123 (22). https://doi.org/10.1063/1.5025856.

(22) Rota, M. B.; Ameruddin, A. S.; Fonseka, H. A.; Gao, Q.; Mura, F.; Polimeni, A.; Miriametro, A.; Tan, H. H.; Jagadish, C.; Capizzi, M. Bandgap Energy of Wurtzite InAs Nanowires. Nano Letters 2016, 16 (8), 5197-5203. https://doi.org/10.1021/acs.nanolett.6b02205.

(23) Bao, J.; Bell, D. C.; Capasso, F.; Erdman, N.; Wei, D.; Fröberg, L.; Mårtensson, T.; Samuelson, L. Nanowire-Induced Wurtzite Inas Thin Film on Zinc-Blende Inas Substrate. Advanced Materials 2009, 21 (36), 3654-3658. https://doi.org/10.1002/adma.200900617.

(24) Möller, M.; De Lima, M. M.; Cantarero, A.; Chiaramonte, T.; Cotta, M. A.; likawa, F. Optical Emission of InAs Nanowires. Nanotechnology 2012, 23 (37), 375704-375737. https://doi.org/10.1088/0957-4484/23/37/375704.

(25) Trägårdh, J.; Persson, A. I.; Wagner, J. B.; Hessman, D.; Samuelson, L. Measurements of 
the Band Gap of Wurtzite InAs 1-XP x Nanowires Using Photocurrent Spectroscopy. Journal of Applied Physics 2007, 101 (12). https://doi.org/10.1063/1.2745289.

(26) De, A.; Pryor, C. E. Predicted Band Structures of III-V Semiconductors in the Wurtzite Phase. Physical Review B - Condensed Matter and Materials Physics 2010, 81 (15). https://doi.org/10.1103/PhysRevB.81.155210.

(27) Faria Junior, P. E.; Campos, T.; Bastos, C. M. O.; Gmitra, M.; Fabian, J.; Sipahi, G. M. Realistic Multiband K.p Approach from Ab Initio and Spin-Orbit Coupling Effects of InAs and InP in Wurtzite Phase. Physical Review B 2016, 93 (23), 1-14. https://doi.org/10.1103/PhysRevB.93.235204.

(28) Gmitra, M.; Fabian, J. First-Principles Studies of Orbital and Spin-Orbit Properties of GaAs, GaSb, InAs, and InSb Zinc-Blende and Wurtzite Semiconductors. Physical Review B 2016, 94 (16), 1-10. https://doi.org/10.1103/PhysRevB.94.165202.

(29) Zanolli, Z.; Fuchs, F.; Furthmüller, J.; Von Barth, U.; Bechstedt, F. Model GW Band Structure of InAs and GaAs in the Wurtzite Phase. Physical Review B - Condensed Matter and Materials Physics 2007, 75 (24), 1-8. https://doi.org/10.1103/PhysRevB.75.245121.

(30) Bechstedt, F.; Belabbes, A. Structure, Energetics, and Electronic States of III-V Compound Polytypes. Journal of Physics Condensed Matter. 2013. https://doi.org/10.1088/0953$8984 / 25 / 27 / 273201$.

(31) Joyce, H. J.; Wong-Leung, J.; Gao, Q.; Hoe Tan, H.; Jagadish, C. Phase Perfection in Zinc Blende and Wurtzite III- V Nanowires Using Basic Growth Parameters. Nano Letters 2010, 10 (3), 908-915. https://doi.org/10.1021/nl903688v.

(32) Alexander-Webber, J. A.; Groschner, C. K.; Sagade, A. A.; Tainter, G.; Gonzalez-Zalba, M. F.; Di Pietro, R.; Wong-Leung, J.; Tan, H. H.; Jagadish, C.; Hofmann, S.; et al. Engineering the Photoresponse of InAs Nanowires. ACS Applied Materials and Interfaces 2017, 9 (50), 43993-44000. https://doi.org/10.1021/acsami.7b14415.

(33) Ullah, A. R.; Joyce, H. J.; Tan, H. H.; Jagadish, C.; Micolich, A. P. The Influence of Atmosphere on the Performance of Pure-Phase WZ and ZB InAs Nanowire Transistors. Nanotechnology 2017, 28 (45). https://doi.org/10.1088/1361-6528/aa8e23.

(34) Adachi, S. Optical Properties of Crystalline and Amorphous Semiconductors; Springer US, 1999. https://doi.org/10.1007/978-1-4615-5241-3.

(35) Pavesi, L.; Piazza, F.; Rudra, A.; Carlin, J. F.; llegems, M. Temperature Dependence of the InP Band Gap from a Photoluminescence Study. Physical Review B 1991, 44 (16), 90529055. https://doi.org/10.1103/PhysRevB.44.9052.

(36) Geng, P.; Li, W.; Zhang, X.; Zhang, X.; Deng, Y.; Kou, H. A Novel Theoretical Model for the Temperature Dependence of Band Gap Energy in Semiconductors. Journal of Physics D: Applied Physics 2017, 50 (40). https://doi.org/10.1088/1361-6463/aa85ad.

(37) O’Donnell, K. P.; Chen, X. Temperature Dependence of Semiconductor Band Gaps. Applied Physics Letters 1991, 58 (25), 2924-2926. https://doi.org/10.1063/1.104723.

(38) Kumar, P.; Wade, A.; Smith, L. M.; Jackson, H. E.; Yarrison-Rice, J. M.; Choi, Y. J.; Park, J. G. Photocurrent Spectroscopy of Single CdS Nanosheets: Valence Band Structure and Two Photon Absorption. Applied Physics Letters 2011, 98 (14), 98-101. https://doi.org/10.1063/1.3573859.

(39) Chuang, S.; Chang, C. K·p Method for Strained Wurtzite Semiconductors. Physical Review B - Condensed Matter and Materials Physics 1996, 54 (4), 2491-2504. 
https://doi.org/10.1103/PhysRevB.54.2491.

(40) Turnbull, David, Ehrenreich, Henry, Seitz, F. Solid State Physics, first.; Elsevier, 1981.

(41) Madelung, O. Semiconductors: Data Handbook, third.; Springer, 2004.

(42) Sun, M. H.; Joyce, H. J.; Gao, Q.; Tan, H. H.; Jagadish, C.; Ning, C. Z. Removal of Surface States and Recovery of Band-Edge Emission in InAs Nanowires through Surface Passivation. Nano Letters 2012, 12 (7), 3378-3384. https://doi.org/10.1021/nl300015w.

(43) Ruda, H. E.; Shik, A. Polarization-Sensitive Optical Phenomena in Semiconducting and Metallic Nanowires. Physical Review B - Condensed Matter and Materials Physics 2005, 72 (11), 1-11. https://doi.org/10.1103/PhysRevB.72.115308.

(44) Ruda, H. E.; Shik, A. Polarization-Sensitive Optical Phenomena in Thick Semiconducting Nanowires. Journal of Applied Physics 2006, 100 (2). https://doi.org/10.1063/1.2216879.

(45) Jensen, B. Quantum Theory of the Complex Dielectric Constant of Free Carriers in Polar Semiconductors. IEEE Journal of Quantum Electronics 1982, 18 (9), 1361-1370. https://doi.org/10.1109/JQE.1982.1071713.

(46) De, A.; Pryor, C. E. Optical Dielectric Functions of Wurtzite III-V Semiconductors. Physical Review B - Condensed Matter and Materials Physics 2012, 85 (12), 1-9. https://doi.org/10.1103/PhysRevB.85.125201.

(47) De Luca, M.; Zilli, A.; Fonseka, H. A.; Mokkapati, S.; Miriametro, A.; Tan, H. H.; Smith, L. M.; Jagadish, C.; Capizzi, M.; Polimeni, A. Polarized Light Absorption in Wurtzite InP Nanowire Ensembles. Nano Letters 2015, 15 (2), 998-1005. https://doi.org/10.1021/nl5038374.

(48) Kim, D. C.; Dheeraj, D. L.; Fimland, B. O.; Weman, H. Polarization Dependent Photocurrent Spectroscopy of Single Wurtzite GaAs/AlGaAs Core-Shell Nanowires. Applied Physics Letters 2013, 102 (14). https://doi.org/10.1063/1.4801865. 


\section{Exploring the Band Structure of Wurtzite InAs Nanowires Using Photocurrent Spectroscopy}

Seyyedesadaf Pournia, Samuel Linser, Giriraj Jnawali, Howard E. Jackson and Leigh M. Smith Department of Physics, University of Cincinnati, Cincinnati, OH 45221-0011

Amira Ameruddin, Philippe Caroff, Jennifer Wong-Leung, Hark Hoe Tan, and Chennupati Jagadish

Department of Electronic Materials Engineering, Research School of Physics, The Australian National University, Canberra, ACT 2601, Australia

Hannah J. Joyce

Department of Engineering, University of Cambridge, 9 JJ Thomson Avenue, Cambridge CB3 oFA, United Kingdom

\section{Supplementary Information}

Photocurrent measurements were taken repeatedly on different days to confirm that the peaks which we are considering as the transitions between the energy bands are reproducible. Some low temperature photocurrent spectra in the energy range of $(0.82-1.24) \mathrm{eV}$ are presented in Figure S1 below.

To identify each transition, we considered the selection rules determined from group theory (Equation 1 in the main text) and energetic order established from the theory in reference 1 to assign the peaks to the transitions. The energy values reported in Table S1 and also in the main text are the average of the energies in figure S1 and the errors are the standard deviations, which are summarized in the table below. 

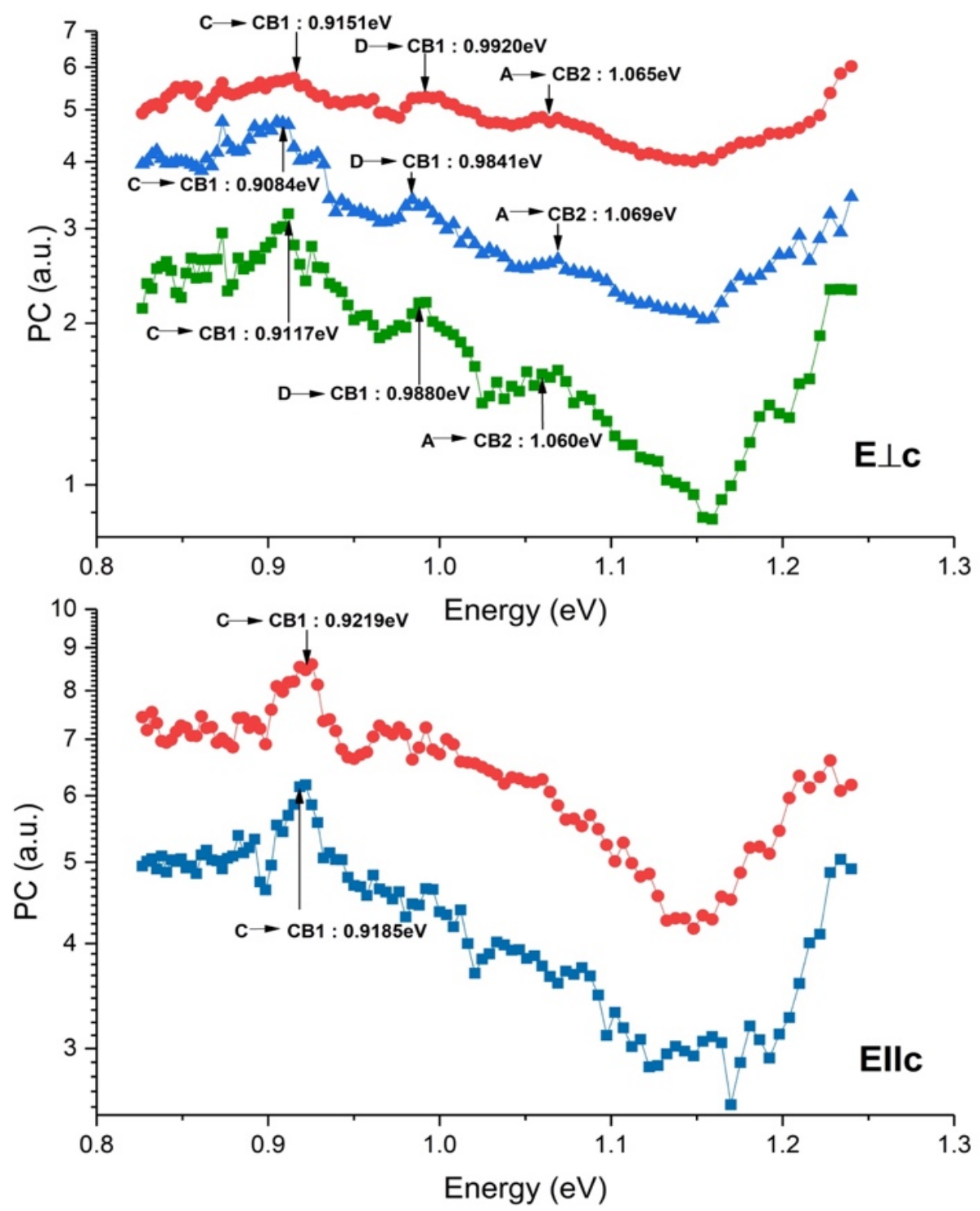

Figure S1. Photocurrent measurements on Wurtzite InAs device at $\mathrm{T}=10 \mathrm{~K}$ with laser excitation polarized perpendicular (top) and parallel (bottom) to the c-axis of nanowire.

Table S1. Average energies and standard deviations.

\begin{tabular}{|l|l|l|}
\hline Transition & Mean $(\mathrm{eV})$ & Standard Deviation $(\mathrm{eV})$ \\
\hline $\mathrm{C} \rightarrow \mathrm{CB} 1$ & 0.915 & 0.005 \\
\hline $\mathrm{D} \rightarrow \mathrm{CB} 1$ & 0.988 & 0.003 \\
\hline $\mathrm{A} \rightarrow \mathrm{CB} 2$ & 1.065 & 0.004 \\
\hline
\end{tabular}




\section{References:}

(1) De, A.; Pryor, C. E. Predicted Band Structures of III-V Semiconductors in the Wurtzite Phase. Physical Review B - Condensed Matter and Materials Physics 2010, 81 (15). https://doi.org/10.1103/PhysRevB.81.155210. 